\title{
A new partially autonomous camera control system
}

\author{
M. Polski ${ }^{1}$, A. Fiolka ${ }^{1}$, S.Can ${ }^{1}$, A.Schneider ${ }^{1}$, H. Feussner ${ }^{1,2}$ \\ ${ }^{1}$ Workgroup MITI, Klinikum rechts der Isar, Technische Universität München \\ ${ }^{2}$ Department of Surgery, Klinikum rechts der Isar, Technische Universität München
}

\begin{abstract}
The minimal invasive surgery is commonly used in clinical practice. The advantages such as less complication rate or shorter recovery time are unquestionable. But the lack of direct touch feedback and a narrow field of view makes this approach difficult for the surgeon to apply. To make the procedure easier, supportive systems are being build. One of the above is the camera control system constructed on the basis of the data from position sensors and an endoscope manipulating robot. Implemented features like speed adjustment or hysteresis ensure intuitive usage. The time necessary for the successful completion of the surgeon's training tasks with an accuracy of $+/-10 \mathrm{~mm}$ decreased. Users gave positive feedback concerning the operation of the system.
\end{abstract}

Keywords - Minimal Invasive Surgery, Camera, Endoscope, Camera Guidance

\section{INTRODUCTION}

In minor access surgery (MAS), an assistant guides the camera and makes the field of surgery visible. This task is stressful and needs full concentration on the part of the whole operating team, especially during long interventions. Misunderstandings are common. Recent research has shown that time loss was mainly a result of the time-consuming positioning of the system and the delicate port placement [1]. This and other limitations lead to interruptions and the surgeon's lack of concentration [2].

A number of support systems have been built to improve this situation [3].They range from very simple manual holding arms [4], through the voice-controlled [5] to very sophisticated and complex image recognition systems.

All these systems have their drawbacks. The mechanical arms are very simple and need manual positioning. Voice recognition algorithms, on the other hand, have to be improved to work reliably, so that constant „chatting“ at the operating table would not disturb the procedure. Systems based on image recognition seem really promising. However, the problem is that during the operation the tracked marker may be covered with blood or adjacent organs.

A camera positioning system based on electromagnetic sensors might provide a possible solution to some of the problems listed above. The SOLOASSIST, a fluid driven robot which produces no electromagnetic disturbance, makes such a system possible to build. This paper discusses the possible design and implementation of the new, partially autonomous camera control system based on the SOLOASSIST robot and the electromagnetic position sensors.

\section{Materials AND Methods}

The proposed system consists of three basic parts:

1. position sensors,

2. control unit

3. mechanic manipulator

MiniBird magnetic tracker, produced by Ascension (Burlington, VT, USA), was used as a tracking device. The endoscope was manipulated by the SOLOASSIST robot (AKTORmed, Barbing, Germany). The control software was implemented into the SOLOASSIST control unit using the ICONNECT development system. (MICRO-EPSILON, Ortenburg, Germany)

At the very beginning, the sensors were tested. They were connected to a minimal-invasive surgical instrument while the control unit was calculating the position of the tip of the instrument.

Then, the accuracy of the setup was examined. The surgical tool, with the sensor attached to its handle, was being directed at different points within the workspace and the control unit calculated the tip position. The data so collected were then compared with real values. All experiments were repeated with two sensors per object (Fig.1). It is thanks to this data that the software controlling the robot could be written.

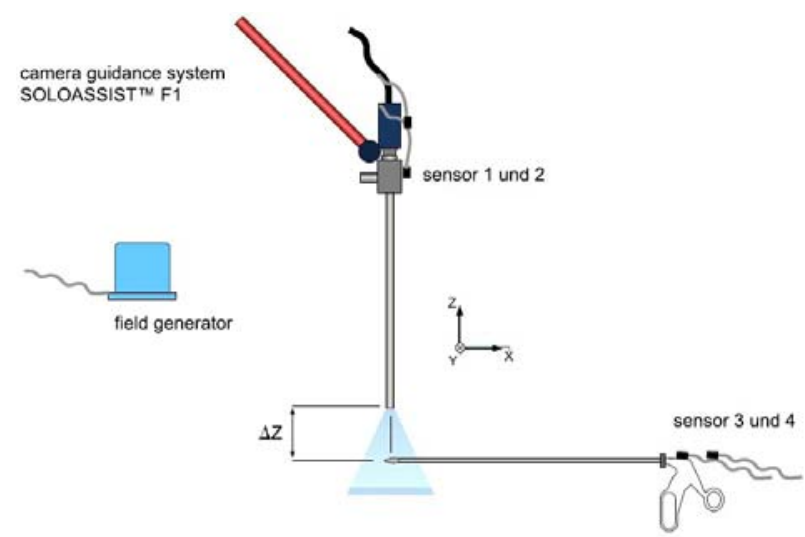

Fig. 1 The draft of the autonomous camera control system 
The main task of the camera control system is to keep the desired distance between the tip of the surgical tool and the endoscope camera, and, in this way, to provide constant view of the operating area. The above procedure, as well as the calibration performed at the beginning of the operation to adjust the desired distance, are being implemented into the control unit.

To improve the performance of the system several improvements were added. For instance, it became possible to switch the operation mode between manual and automatic at any time. Speed adjustment was introduced to make the system work more intuitively. Control algorithm was improved by the implementation of hysteresis to reduce the small, disturbing movements of the camera. The control unit was made to react only to normal movements and not to noise or surgeon's hand trembling.

Finally, the precision was measured. The implemented, partially autonomous camera control system was tested and the results were compared with the standard approach. The next step taken by our team was participation in a training task for surgeons, where a needle had to be passed through 2-mm long rings mounted on a plate.

\section{ResUltS}

The autonomous camera control system has been successfully realized. The newly developed system receives the position and orientation of the tip of the instrument from the tracking module and uses this information to position the endoscope.

The precision measurements were carried out in the following way. The plate with circles of diameter ranging from 10 to $150 \mathrm{~mm}$ was mounted on the bottom of the test box. The tip was pointed at the circles at certain angles. Then, the output from endoscope camera was examined and the distance between the tool's tip and the center of the screen was measured. The results may be found in Table 1.

The precision in the circles of diameter up to $100 \mathrm{~mm}$ long appears within the range of $\pm 10 \mathrm{~mm}$ and then it increases. Nevertheless, the tip of the instrument is always visible for the endoscope camera. Error data presented in Table 1 might seem very large, yet, in reality, owing to the implemented hysteresis, they do not affect the performance. To put it simply, the system does not perceive the small disturbing movements. To change the camera position the surgeon moves the instrument in a desired direction and the system follows this movement. The task can then be completed without camera movement. The accuracy, resulting from hysteresis set in the control panel, might seem a weakness, but it turns out to be a great advantage. Some members of our team have found the operation with relatively large hysteresis very convenient.

The intuitive performance of the system is possible thanks to the speed adjustment. The smaller the distance to cover, the slower the robot's movements. All the above results seem very promising for the SOLOASSIST control unit.

Table 1 EVM measurments table

\begin{tabular}{|c|c|c|c|c|c|c|c|c|}
\hline \multicolumn{9}{|c|}{ EVM $[\mathrm{mm}]$ for circles of fixed diameter } \\
\hline diameter $[\mathrm{mm}]$ & 10 & 30 & 50 & 70 & 90 & 110 & 130 & 150 \\
\hline $0^{\circ}$ & 15 & 4 & 24 & 13 & 12 & 19 & 24 & 30 \\
\hline $30^{\circ}$ & & & 14 & 18 & 7 & 18 & 19 & 18 \\
\hline $60^{\circ}$ & & 11 & 9 & 16 & 11 & 11 & 16 & 19 \\
\hline $90^{\circ}$ & 18 & & 8 & 9 & 10 & 11 & 4 & 15 \\
\hline $120^{\circ}$ & & 13 & 20 & 5 & 13 & 24 & 26 & 21 \\
\hline $150^{\circ}$ & & & 19 & 16 & 29 & 24 & 50 & 48 \\
\hline $180^{\circ}$ & 5 & 13 & 15 & 15 & 13 & 36 & 25 & 21 \\
\hline $210^{\circ}$ & & & 22 & 24 & 20 & 27 & 34 & 57 \\
\hline $240^{\circ}$ & & 16 & 2 & 5 & 25 & 29 & 33 & 39 \\
\hline $270^{\circ}$ & 9 & & 12 & 17 & 10 & 27 & 13 & 25 \\
\hline $300^{\circ}$ & & 11 & 11 & 15 & 15 & 15 & 19 & 30 \\
\hline $330^{\circ}$ & & & 3 & 5 & 17 & 19 & 19 & 26 \\
\hline \multicolumn{9}{|c|}{ mean value } \\
\hline & 12 & 11 & 13 & 13 & 15 & 21 & 24 & 29 \\
\hline
\end{tabular}

\section{DisCuSION}

Irrespective of its development, the system still needs to be improved. The new tracking sensors should be tested. It may also be useful to combine the electromagnetic system with an image-recognition software or distance measuring sensors.

The experiments confirmed that an electromagnetic tracking system is a potential alternative to other positioning techniques. It works reliably, silent, and insensitive to endoscope image disturbance.

\section{REFERENCES}

[1] J. Bodner, R. Kafka-Ritsch, P. Lucciarini, and T. Schmid. A Critical Comparison of Robotic Versus Conventional Laparoscopic Splenectomies. World Journal of Surgery, 29(8):982-986, 2005.

[2] G.B. Cadiere. Feasibility of Robotic Laparoscopic Surgery: 146 Cases. World Journal of Surgery,

[3] D.B. Camarillo, T.M. Krummel, and J.K. Salisbury. Robotic technology in surgery: Past, present, and future. The American Journal of Surgery, 188(4S1):2-15, 2004.

[4] A. Arezzo, A. Braun, G. F. Buess, and M. O. Schurr. Experimental assessment of a new mechanical endoscopic solosurgery system: Endofreeze,. Surgical Endoscopy, 19(4):581-588, 2005.

[5] G. H. Ballantyne. Robotic surgery, telerobotic surgery, telepresence, and telementoring,. Surgical Endoscopy, 16(10):1389-1402, 2002.

\section{Corresponding Author:}

Hubertus Feussner, MD

Technische Universität München

Klinikum rechts der Isar

Department of Surgery

Ismaninger Str. 22, 81675 München

Tel.: +49 89 4140-0, Fax +49 89 4140-0

E-Mail: Feussner@chir.med.tu-muenchen.de 